\title{
Estado nutricional e hábitos alimentares de acadêmicos de uma universidade do norte do Rio Grande do Sul
}

\author{
Andressa Carine Kretschmer ${ }^{1}$, Géssica de Oliveira Rodrigues², \\ Andressa dos Santos Ristow², Nilce Coelho Peixoto ${ }^{3}$
}

\begin{abstract}
RESUMO
O objetivo da presente pesquisa foi identificar a relação entre o estado nutricional e hábitos alimentares e prática de atividade física de jovens acadêmicos. Tratou-se de um estudo transversal, no qual utilizou-se variáveis antropométricas e questionário sobre hábitos alimentares e estilo de vida com os graduandos das mais diversas áreas da UFSM/CESNORS, Palmeira das Missões com faixa etária de 17 a 24 anos. Os resultados observados no estudo revelam que a amostra tem uma considerável parcela de indivíduos com a constituição corporal inadequada, 26,4\% estava com o índice de massa corporal (IMC) acima do preconizado e 30,19\% estava com o percentual alto de gordura. Associado a isto, têm hábitos alimentares pouco saudáveis, pratica pouca atividade física e ingere bebidas alcoólicas com frequência. Os presentes dados merecem destaque, uma vez que o desenvolvimento de muitas doenças crônicas se dá em decorrência dos maus hábitos e acúmulo excessivo de gordura.
\end{abstract}

Descritores: Jovens; Estado Nutricional; Hábitos Alimentares.

\section{Nutritional status and feeding habits of college of a northern university of Rio Grande do Sul}

\begin{abstract}
The objective of this research was to identify the relationship between nutritional status and feeding habits and physical activity of young colleges. The experimental subjects were young academics aged between 17 a 24 years-old, students from various undergraduate courses UFSM of Palmeira das Missões/RS, which underwent anthropometric measurements and completed a questionnaire about their habits. The results observed in the current study reveal that the studied sample has a considerable number of individuals with inadequate body constitution, $26.4 \%$ of the study population was the body mass index (BMI) was above the recommended, and is $30.19 \%$ with the percentage body fat rated as below average (worse than average), bad and very bad. Additonally, have unhealthy eating habits, lack of physical activity practice and routinely ingests alcohol. The present data are noteworthy, because the principle of developing of many chronic diseases are the result of bad habits and accumulation of excessive fat.
\end{abstract}

Descriptors: Young; Nutritional Status; Food Habits.

${ }^{1}$ Graduada em Nutrição pela Universidade Federal de Santa Maria (UFSM), Palmeira das Missões, RS, Brasil.

${ }^{2}$ Acadêmica em Nutrição na Universidade Federal de Santa Maria (UFSM), Palmeira das Missões, RS, Brasil.

${ }^{3}$ Doutora em Bioquímica Toxicológica pela Universidade Federal de Santa Maria (UFSM), Docente do CESNORS, UFSM, Palmeira das Missões, RS, Brasil. 


\section{Introdução}

As mudanças ocorridas nos últimos anos, com o predomínio do consumo de alimentos hipercalóricos associado à falta de atividade física, tem levado a maior parte da população a apresentar maior acúmulo de gordura e consequente aumento de peso corporal ${ }^{1-2-3}$. A população jovem é bastante afetada por estas mudanças uma vez que se encontra naturalmente mais ligada à mídia e sob sua influência'.

O consumo de alimentos ricos em gorduras e açúcares, assim como a influência da família, ambiente e amigos favorece 0 aparecimento da obesidade e sobrepeso ${ }^{4}$. Sabe-se que alterações alimentares e no estilo de vida possuem ligação com a incidência de doenças crônicas não transmissíveis. $O$ controle de peso corporal dentro dos parâmetros da eutrofia e a manutenção de hábitos alimentares saudáveis, assim como outros fatores, é importante no desenvolvimento de algumas patologias 5 .

$\mathrm{O}$ ingresso na universidade, muitas vezes acompanhado da mudança para outro município e do fato de passar a morar sozinho ou com colegas e amigos (desacompanhado da família), acarreta em alterações nesta fase da vida e são marcadas por influências psicológicas, sociais e econômicas, que, muitas vezes, alteram os padrões de hábitos alimentares de muitos universitários ${ }^{6}$. Os maus hábitos alimentares dos estudantes, como lanches de preparação rápida, com excesso de calorias ou calorias vazias, estão ligados às situações de estresse físico e mental vivenciados ${ }^{7}$.

Os hábitos dietéticos, estilo de vida, perfil lipídico e conformação corporal passa a ter importância somente na vida adulta, pois o diagnóstico ou o aparecimento das doenças crônicas, na maior parte das vezes, se dá nesta fase ou na idade senil, mediante o acúmulo excessivo de lipídios séricos, iniciado na juventude, ou mesmo através de outras doenças crônicas decorrentes do excesso de peso, tais como diabetes e hipertensão arterial? .

Os hábitos alimentares iniciados durante o período universitário mantêm-se na idade adulta. Portanto, caracterizar os hábitos e a conformação corporal desta população é de extrema importância. $O$ excesso de peso na juventude, assim como o acúmulo demasiado de gordura merece destaque, pois quanto mais cedo diagnosticados, os possíveis agravos podem ser evitados ${ }^{9}$.

A prevenção, iniciada precocemente (no período jovem da vida), assim como o tratamento não farmacológico, a fim de evitar os altos índices colesterolêmicos e as doenças crônicas, baseia-se em modificações do estilo de vida, através da adoção de hábitos alimentares saudáveis, associados com a prática de exercícios físicos regulares ${ }^{10}$.

Em virtude disto, a presente pesquisa teve por finalidade identificar a prevalência e os fatores associados ao percentual de gordura elevado e ao excesso de peso em uma amostra de universitários da Universidade Federal de Santa Maria (UFSM), Centro de Educação Superior Norte (CESNORS), na cidade de Palmeira das Missões, Rio Grande do Sul, Brasil, pois entende-se que conhecer os fatores de risco para o desenvolvimento de doenças crônicas é de fundamental importância para que possa ser avaliada a necessidade de projetos em prol da saúde desta população.

\section{Metodologia}

Trata-se de um estudo transversal, com amostra de conveniência, com a finalidade de identificar a prevalência de maus hábitos alimentares e estilo de vida, sobrepeso, obesidade e percentual de gordura corporal elevado em uma amostra de acadêmicos da UFSM, Campus de Palmeira das Missões. Para o estudo elencaram-se estudantes de ambos os sexos, de 17 a 24 anos, que voluntariamente dispuseram-se para a realização do mesmo.

Para a concretização desta pesquisa foi elaborado e utilizado um questionário contendo perguntas semi-abertas e fechadas, onde os participantes tiveram a oportunidade de se expressar, revelando aspectos subjetivos que não seriam encontrados em outras fontes, tais como a frequência de consumo de frutas, verduras, substituição do almoço ou jantar por lanches, ingesta de bebida alcoólica, nível de atividade física e outros hábitos. Os critérios adotados na avaliação do consumo alimentar foram aqueles preconizados pelo Guia alimentar para a população brasileira: promovendo a alimentação saudável11. Os voluntários foram abordados nas salas de aula e os questionários respondidos neste mesmo ambiente.

Os dados relativos ao IMC e às dobras cutâneas foram coletados numa sala privativa, localizada no Campus da UFSM. A coleta dos mesmos foi feita por acadêmicas do curso de graduação em Nutrição treinadas para tal finalidade.

Para o cálculo do IMC, coletou-se a altura e o peso corporal do participante. A coleta de medidas realizou-se com 0 mínimo de vestimentas leves e sem calçados. Para aferir a altura, fez-se de maneira ereta, com o olhar para o infinito, a fita métrica foi fixada em uma parede sem desníveis e o indivíduo esteve de pernas fechadas e com o calcanhar encostado na parede. Para aferir o peso, utilizou-se balança eletrônica, com capacidade para até $200 \mathrm{~kg}$, admitindo-se 
variação de, no máximo, $200 \mathrm{~g}$ entre duas medidas. O IMC, para os indivíduos com idade superior a 20 anos, foi obtido através de sua respectiva equação: peso (em $\mathrm{kg}$ ) dividido por altura $(\mathrm{em} \mathrm{m})$ ao quadrado e classificou-se os indivíduos conforme os parâmetros da Organização Mundial da Saúde ${ }^{12}$. Para os indivíduos menores de 19 anos, a classificação procedeu-se conforme 0 que preconiza os escores para a respectiva faixa etária ${ }^{13}$.

Para a avaliação das dobras cutâneas, em mulheres coletou-se a espessura das pregas cutâneas tricipital, suprailíaca e abdominal e, em homens, a torácica, subescapular e tricipital com o uso de plicômetro científico. Considerou-se a média de três aferições consecutivas, excluindo-se os valores cuja diferença foi superior a $1 \mathrm{~mm}$ entre uma medida e outra. Adotaramse os métodos de aferição quanto à localização das pregas, sendo a dobra cutânea abdominal aferida a três centímetros da cicatriz umbilical e a um centímetro abaixo do centro da cicatriz umbilical. A dobra suprailíaca foi coletada em cima da linha axilar média e logo acima da crista ilíaca. A dobra tricipital, no ponto médio da região posterior do braço, a dobra subescapular foi localizada no sentido diagonal no ângulo inferior da escápula e a torácica, no ponto médio entre a linha axilar anterior e o mamilo ${ }^{14}$. A densidade corpórea foi determinada através de cálculos específicos para os sexos feminino e masculino ${ }^{15-16}$.

Posteriormente, obteve-se o percentil de gordura corporal pela fórmula de SIRI G\% $=(4,95 /$ Densidade Corporal) $-4,50$ $x 100^{17}$. Classificaram-se os valores de percentil de gordura conforme idade e sexo com as devidas classificações ${ }^{18}$.

Foi observado rigorosamente o que consta na Resolução 466/12 do Ministério da Saúde. 0 projeto foi aprovado pelo Comitê de Ética em Pesquisa da UFSM sob o número de protocolo 555.166, em 13 de março de 2014. Os acadêmicos que concordaram em fazer parte do estudo leram e assinaram o Termo de Consentimento Livre Esclarecido (TCLE) previamente ao início dos trabalhos de coleta de dados.

Os dados foram tabulados em uma planilha do programa Excel 2010. Os resultados de IMC e percentual de gordura foram categorizados segundo a OMS (2006 e 2007 $)^{12-13}$ e Pollock e Wilmore (1993) $)^{18}$, respectivamente. Para os resultados oriundos do questionário, foram calculadas as frequências percentuais.

\section{Resultados e Discussão}

Neste trabalho foram avaliados 53 acadêmicos de cursos de graduação da UFSM, Campus Palmeira das Missões, onde há 1.233 estudantes. Destes, 14 eram do sexo masculino e 39, do sexo feminino. A média de idade do grupo foi de 20,45 \pm 1,87 anos (média \pm desvio padrão), sendo que o grupo feminino teve média de 20,00 $\pm 1,65$ anos e 0 grupo masculino, média de 21,71 $\pm 1,90$ anos. A amostra estava constituída de 17 indivíduos que eram adolescentes (menores de 20 anos de idade), o que perfaz $32 \%$. Onze participantes (20,75\%) eram fumantes e os demais, 42 (79,25\%), eram não fumantes. Foi verificado que $100 \%$ dos fumantes pertenciam ao sexo feminino. $O$ consumo de tabaco merece destaque, pois o mesmo está associado à gênese de muitas doenças crônicas, tais como hipertensão, aterosclerose, cânceres e doenças respiratórias ${ }^{19}$. Conforme dados da OMS, referente a 151 países, estima-se que $7 \%$ de meninas adolescentes fumam, contra $12 \%$ dos meninos ${ }^{20}$. A discrepância observada em relação ao fato de que todos os tabagistas da amostra eram do sexo feminino comparativamente aos dados publicados pela OMS pode ser atribuída ao fato de que a maioria dos cursos de graduação oferecidos neste campus desta instituição são predominantemente frequentados pela clientela feminina e também devido a amostra ser constituída por cerca de um quarto de mulheres.

Quanto ao hábito de ingerir bebidas alcoólicas, cerca de $40 \%$ dos participantes (21 acadêmicos) respondeu que bebe semanalmente. Sessenta por cento ingeria álcool esporadicamente ou nunca. Cabe ressaltar que as bebidas alcoólicas possuem elevado valor calórico, portanto, seu consumo excessivo tem ligação direta com o aumento do peso corporal, adicionalmente, dependendo da frequência e do modo de consumo ${ }^{22}$.

Quando indagados sobre a prática de atividade física, 45,28\% (24 sujeitos) declararam que praticam atividade física por mais de 30 minutos de uma a quatro vezes por semana. Apenas 5,66\% (3 indivíduos do sexo feminino) declararam-se muito ativos, praticando atividade física diariamente. Aproximadamente a metade dos indivíduos (47,17\%) jamais se exercita ou raramente o faz. 0 exercício pode ter impacto sobre a ingesta calórica inadequada e sobre o estresse exagerado. No controle do peso corporal, o mesmo é muito importante, uma vez que indivíduos que se exercitam possuem uma demanda energética maior, comparada aqueles que não o fazem ${ }^{21}$. Um estudo realizado com 281 estudantes de cursos da área da saúde na Universidade de Brasília evidenciou que $79,7 \%$ dos estudantes apresentavam inadequações nos hábitos alimentares e 65,5\% eram sedentários, não se incluíram nestes resultados os acadêmicos do curso de Educação Física. Estes dados preocupam, uma vez que os estudantes da área da saúde serão futuros disseminadores de hábitos saudáveis ${ }^{23}$. 
Cerca de um terço dos respondentes (35,85\%) alegou nunca ter feito a dosagem sanguínea de colesterol. Dentre os homens, mais do que a metade deles $(57,14 \%)$ jamais foi submetido a este exame, mas entre o público feminino este percentual foi de $28,20 \%$. Investigações já apontam a incidência do colesterol elevado em fases precoces da vida. Em um estudo transversal com 374 crianças de 10 a 12 anos de idade, de escolas das redes pública e privada, realizado na cidade de Santa Maria-RS, foi constatado que as prevalências de hipercolesterolemia, hipertrigliceridemia e excesso de peso encontrado foram de 4,7\%,8,9\% e 20,7\%, respectivamente. As crianças com excesso de peso apresentaram maior prevalência de alterações lipídicas ${ }^{24}$. $O$ alto teor de colesterol nas idades iniciais e na juventude está relacionado, na maior parte dos casos, à má alimentação e ao sedentarismo. 0 consumo exagerado de lipídios pode elevar o nível de colesterol no sangue e o mesmo não traz problemas imediatos. Porém, quando sem tratamento, aumenta o risco de doenças cardíacas na fase adulta e senil. Para tanto, quantificar os índices colesterolêmicos e detectar possíveis dislipidemias pode prevenir consequentes agravos ${ }^{24-25}$.

$\mathrm{O}$ questionamento sobre o consumo diário de legumes ou de verduras mostrou que existe uma ingestão adequada deste tipo de alimento entre a maioria dos participantes (60,38\%). Entretanto, cerca de um quinto deles não consome estes alimentos, o que coincide com o consumo de frutas no dia a dia. A ingestão diária de frutas foi considerada ideal somente em $5,66 \%$ da amostra, sendo que este percentual foi representado exclusivamente pelo grupo feminino. Segundo o "Guia Alimentar para a População Brasileira: promovendo a alimentação saudável", elaborado pelo Ministério da Saúde, recomenda-se o consumo de três porções de frutas e três porções de legumes e verduras diariamente ${ }^{11}$.

Aingestão de carne vermelha ou de frango é acompanhada da gordura aparente para um terço dos respondentes. Foi observado que entre os homens há um predomínio da ingesta desta gordura associada às carnes $(71,43 \%)$. A substituição de uma refeição convencional (almoço ou jantar) por lanches foi verificada em $79,25 \%$ da população estudada. Quarenta e três por cento dos acadêmicos comem diariamente ou muito frequentemente alimentos do tipo embutidos, frituras, doces e lanches rápidos. As mudanças nos padrões alimentares nas últimas décadas, como o aumento do consumo de açúcares simples, alimentos industrializados com alta densidade energética, alimentos ricos em gorduras e ingestão insuficiente de frutas e hortaliças, contribuem diretamente para o ganho de peso ${ }^{26}$. Considerando que o público estudado é exclusivamente de graduandos jovens, sugere-se que tenham passado recentemente por significativas mudanças de variados tipos, econômicas, de convivência social, de responsabilidade, de consumo alimentar, entre outras, o que pode acarretar em alterações no padrão de hábitos comportamentais e dietéticos.

$O$ índice de massa corpórea (IMC) é um indicador do estado nutricional, porém, o mesmo não classifica a composição corporal do indivíduo em massa magra e massa gorda ${ }^{27}$. Um método indireto de avaliação de gordura corporal trata-se da medida das dobras cutâneas, o qual utiliza equações para o cálculo do percentual, onde há a relação de gordura subcutânea e a densidade corporal, um método eficiente, porém, salienta-se que necessita de treinamento de seus aplicadores no manuseio do adipômetro ${ }^{14}$. Foi encontrado $71,26 \%$ dos indivíduos classificados como eutróficos, um quarto possuía sobrepeso e somente 1 sujeito estava desnutrido (Gráfico 1). Os indivíduos diferem-se em relação à composição corporal e localização da gordura, para tanto, para uma análise mais eficaz, o uso do IMC deve estar associado às medidas de distribuição de gordura corporal².

Constatou-se, na presente investigação, o predomínio de indivíduos com o percentual de gordura classificado como acima da média (melhor que a média). Cerca de um quarto dos participantes estavam com o seu percentual de gordura classificado como bom, dezessete por cento da amostra, situava-se com o percentual dentro da média, 13\% estavam com o percentual abaixo da média (pior do que a média) e sete indivíduos com o percentual ruim. Apenas dois indivíduos da amostra apresentaram percentual de gordura na classificação de muito ruim (Gráfico 2).

Os resultados deste estudo mostrou que os percentuais de adiposidade corporal ideal e excelente somam $69,80 \%$ (somatório do estado excelente, bom, acima da média e média) e o IMC considerado adequado estava presente em $71,69 \%$ dos indivíduos, o que revela extrema similaridade dos resultados obtidos entre os métodos empregados para avaliar o estado nutricional dos sujeitos estudados. 


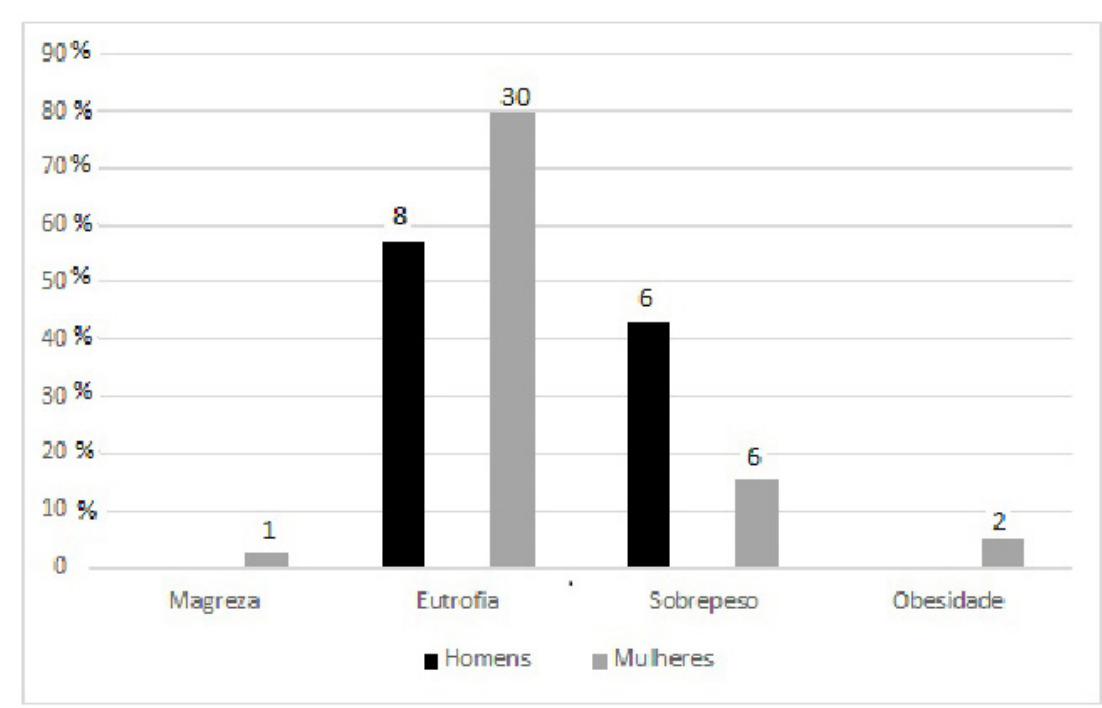

Gráfico 1 - IMC de acadêmicos do CESNORS-PM/UFSM. Classificação segundo a OMS (2007 e 2008)

Vários trabalhos têm demonstrado que a população brasileira, independentemente da idade, apresenta uma tendência para o sobrepeso e obesidade. Em um estudo realizado durante o ano de 2011, na Universidade do Noroeste do Estado do Rio Grande do Sul com 31 estudantes dos cursos de Ciências Biológicas, Pedagogia, Nutrição, e Educação Física foi constatado que $29 \%$ destes estudantes encontravam-se com sobrepeso ${ }^{28}$. Estudantes do nível fundamental, de 9 a 18 anos de idade, da cidade de Bento Gonçalves - RS, apresentaram prevalência de excesso de peso em $24,6 \%$ dos 590 avaliados, sedentarismo em $52,3 \%$, consumo de fast foods por quatro ou mais vezes por semana em $70,3 \%$, dados que preocupam, uma vez que o excesso de peso na infância aumenta as chances da ocorrência de doenças cardíacas na fase adulta ${ }^{29}$. Outra pesquisa realizada com 424 crianças de 6 a 8 anos de idade em Santa Maria- RS, demonstrou a prevalência de obesidade em 29,95\%, conforme o IMC preconizado para a respectiva idade ${ }^{30}$.

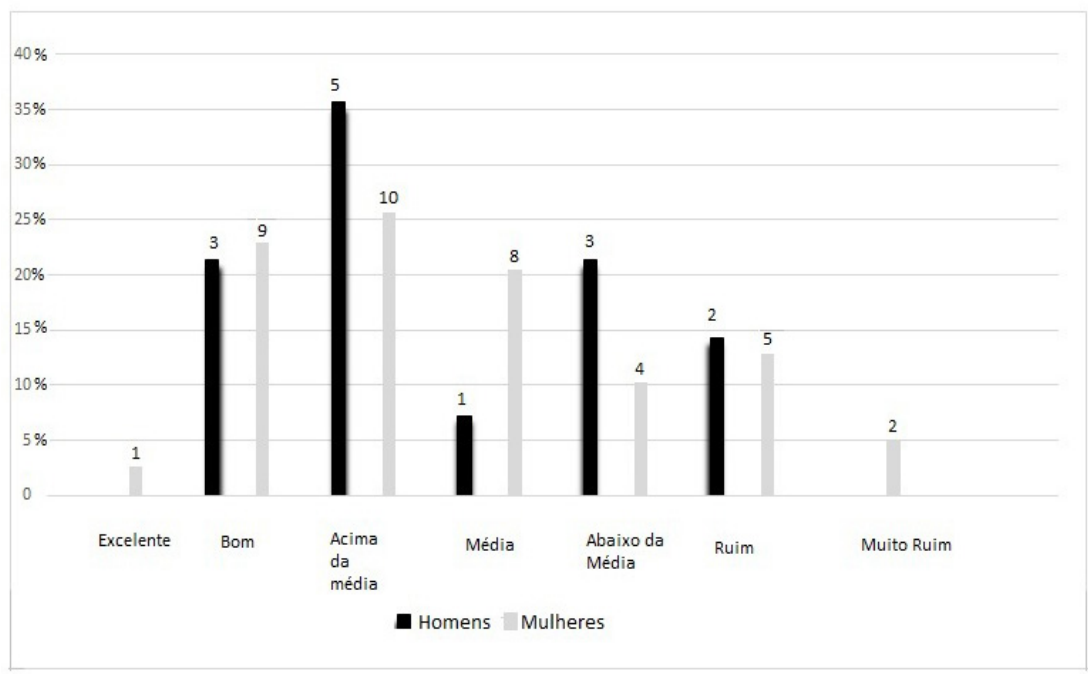

Gráfico 2 - Percentual de gordura corporal de acadêmicos do CESNORS-PM/UFSM. Classificação segundo POLLOCK \& WILLMORE (1993)

Os resultados observados no atual estudo revelam que a amostra estudada tem uma considerável parcela de indivíduos com a constituição corporal inadequada, prevalecendo o excesso de gordura. Associado a isto, têm hábitos alimentares pouco saudáveis, pratica pouca atividade física e ingere bebidas alcoólicas costumeiramente. Embora os indivíduos avaliados estejam numa faixa etária jovem, a manutenção destes fatores propiciará consequências danosas decorrentes disto, uma vez que estudos recentes têm demonstrado que esta população está propensa, em um futuro próximo, a sofrer de patologias associadas ao acúmulo de gordura corporal-9-25-29.

O sobrepeso, caracterizado por quem tem o percentual acima de $25 \mathrm{~kg} / \mathrm{m}^{2}$ de IMC, pode não representar tanto risco à saúde. Entretanto, com o passar do tempo, pode evoluir para a obesidade ${ }^{31}$. Embora, uma quantidade de gordura seja 
essencial para o funcionamento fisiológico, a qual é armazenada em pequenas quantidades na medula óssea, coração, pulmão, fígado, baço, rins, músculos e outros tecidos ${ }^{31}$, o acúmulo excessivo de gordura, consequência do balanço energético positivo, na maior parte das vezes é desencadeada por fatores sócio-ambientais ${ }^{32}$ e merece atenção, pois, na atualidade, as doenças cardiovasculares representam a principal causa de morte em nosso país e os fatores que predispõem o indivíduo são o excesso de peso, o sedentarismo, o tabagismo e 0 alto consumo de gorduras ${ }^{2-3-33-34}$.

\section{Considerações Finais}

Os presentes resultados apontam que a amostra avaliada pratica pouca atividade física, consome álcool frequentemente e uma quantidade de frutas que não é o ideal, substitui com muita frequência uma refeição saudável por alimentos cuja ingestão deve ser muito limitada. Os resultados da coleta antropométrica constataram que existe um número significativo de indivíduos que se encontra com o IMC acima dos parâmetros preconizados para a eutrofia e com o percentual de gordura elevado. Para tanto, deve-se investir em políticas em prol do incentivo da melhoria das condições de saúde desta população, para que, assim, evite-se agravos consequentes de doenças crônicas não transmissíveis, que, na maior parte das vezes, são desencadeados pelos fatores avaliados.

\section{Referências Bibliográficas}

1. Cortese, RDM, Baratto I. Perfil nutricional, alimentar e influência da televisão entre adolescentes de uma escola pública do município de Guarapuava - Paraná. 2010. 28 p. Monografia (conclusão de curso)- Universidade Estadual do Centro-Oeste, 2010. 2. Castro LCV, Franceschini SCC, Priore SE, Pelúzio MCG. Nutrição e doenças cardiovasculares: os marcadores de risco emadultos. Rev Nutr. [periódico na internet]. 2004 Set[citado 2014 Maio 15]; 17(3):369-77. Disponível em: http://www.scielo. br/scielo.php?script=sci_arttext\&pid=S1415-52732004000300010\&Ing=en.doi:10.1590/S1415-52732004000300010.

3. Seki MO, Matsuo T, Seki M. Colesterol não-HDL em escolares de 7 a 17 anos de idade em um município brasileiro. Rev Panam Salud Publica [periódico na internet]. 2007 Maio [citado 2014 Maio 12]; 21(5): 307-12. Disponível em: http:// www.scielosp.org/scielo.php?script=sci_arttext\&pid=S1020-49892007000400006\&lng=en.

doi:10.1590/S1020-49892007000400006.

4. Souza DP. Avaliação do estado nutricional e consumo alimentar de acadêmicos do curso de nutrição da Universidade Federal de Pelotas. Rev HCPA. 2012;32(3):275-82.

5. Veras RP. Estratégias para o enfrentamento das doenças crônicas: um modelo em que todos ganham. Rev Bras Geriatr Gerontol. [periódico na internet]. 2011 [citado 2014 Maio 12];14(4): 779-86. Disponível em: http://www.scielo.br/ scielo.php?script=sci_arttext\&pid=S1809--98232011000400017\&lng=en. doi: 10.1590/S1809-98232011000400017.

6. Souza LB, Malta MB, Donato PB, Corrente JB, Carvalhaes MABL, Papini SJ. Inadequação de consumo alimentar, antropometria e estilo de vida de universitárias da área de saúde. J Health Sci Inst. 2012;30(4):377-81.

7. Vieira VCR, Priore SE, Ribeiro SMR, Franceschini SCC, Almeida LP. Perfil socioeconômico, nutricional e de saúde de adolescentes recém-ingressos em uma universidade pública brasileira. Rev Nutr. [periódico na internet]. 2002 Set [citado 2014 Maio 12];15(3): 273-82. Disponível em: http://www.scielo.br/scielo.php?script=sci_arttext\&pid=S141552732002000300003\&lng=en. doi:10.1590/S1415-52732002000300003.

8. Gerber ZRS, Zielinsky P. Fatores de risco de aterosclerose na infância. Um estudo epidemiológico. Arq Bras Cardiol. [periódico na internet]. 1997 Out [citado 2014 Maio 12]; 69(4): 231-36. Disponível em: http://www.scielo.br/scielo. php?script=sci_arttext\&pid=S0 066-782X1997001000002\&Ing=en. doi: 10.1590/S0066-782X1997001000002.

9. Enes CC, Slater B. Obesidade na adolescência e seus principais fatores determinantes. Rev Bras Epidemiol. [periódico na internet]. 2010 Mar [citado 2014 Maio 12]; 13(1): 163-71. Disponível em:

http://www.scielo.br/scielo.php?script=sci_arttext\&pid=S1415-790X2010000100015\&lng=en.

doi: 0.1590/S1415-790X2010000100015.

10. Rodrigues FM, Petruzellis EA, Bock PM. Avaliação do perfil de usuários de hipolipemiantes de uma drogaria em Porto Alegre-RS, Rev Saúde, 2013; 39(2):149-60. 
11. Ministério da Saúde (BR). Secretaria de Atenção à Saúde. Coordenação-Geral da Política de Alimentação e Nutrição. Guia alimentar para a população brasileira: promovendo a alimentação saudável. Brasília. Ministério da Saúde, 2014. 12. World Health Organization. Global Database on Body Mass Index, 2006. [citado em: 12 de maio 2014].

Disponivel em: <http://apps.who.int/bmi/index.jsp>.

13. World Health Organization. BMl for age (5-19). 2007. [citado 2015 Julho 02]. Disponível em: < http://www.who.int/ growthref/who2007_bmi_for_age/en/>

14. Machado AF. Dobras Cutâneas: localização e procedimentos. Revista de Desporto e Saúde. 2007. 4(2): 41-45.

15. Pollock ML, Loughridge EE, Coleman B, Linnerud AC, Jackson A. Predition of body density in young and middle-aged women. J Appl Physiol. 1975; 38(4): 745-749.

16. Pollock ML, Hickman T, Kendrick Z, Jackson A, Linnerud AC, Dawson G. Prediction of body density in young and middle-aged men. J Appl Physiol 1976;40: 300-304.

17. Siri WE. Body composition from fluids spaces and density: Analyses of methods. In: Techniques for measuring body composition; 1961. 223-224; Washington, DC: National Academy of Science and Natural Resource Council; 1961. p. 223-44. 18. Pollock, ML, Wilmore, JH. Exercícios na Saúde e na Doença: Avaliação e Prescrição para Prevenção e Reabilitação. Rio de Janeiro(RJ): Ed Médica e Científica; 1993.

19. Berto SJP, Carvalhaes MABL, Moura EC. Tabagismo associado a outros fatores comportamentais de risco de doenças e agravos crônicos não transmissíveis. Cad. Saúde Pública [periódico na Internet]. 2010 Ago [citado 2014 Maio 19]; 26(8): 1573-82. Disponível em: http://www.scielo.br/scielo.php?script=sci_arttext\&pid=S0102-311X2010000800011\&lng=en. doi: 10.1590/S0102-311X2010000800011.

20. World Health Organization [página na Internet]. Geneva: World Health Organization [citado 2014 Maio 14]. World No Tobacco Day 2010 - Theme: Gender and tobacco with an emphasis on marketing to women. Disponível em: http://www. who.int/tobacco/wntd/2010/announcement/en/index.html

21. Stein R. Atividade física e saúde pública. Rev Bras Med Esporte [periódico na Internet]. 1999 Ago [citado 2014 Maio 19]; 5(4): 147-49. Disponível em: http://www.scielo.br/scielo.php?script=sci_arttext\&pid=S1517-86921999000400006\&lng=en. doi: 10.1590/S1517-86921999000400006.

22. Kachani AT, Brasiliano S, Hochgraf PB. O impacto do consumo alcoólico no ganho de peso. Rev psiquiatr. clín. [periódico na Internet]. 2008 [citado 2014 Maio 19]; 35(1): 21-24. Disponível em: http://www.scielo.br/scielo.php?script=sci_ arttext\&pid=S0101-60832008000700006\&lng=en. doi:10.1590/S0101-60832008000700006.

23. Marcondelli P, Costa THM, Schmitz BAS. Nível de atividade física e hábitos alimentares de universitários do $3^{\circ}$ ao $5^{\circ}$ semestres da área da saúde. Rev Nutr. [periódico na internet]. 2008. Fev [citado 2014 Maio 12]; 21(1): 39-47.

Disponível em: http://www.scielo.br/scielo.php?script=sci_arttext\&pid=S14115-52732008000100005\&lng=en. doi:10.1590/ S1415-52732008000100005.

24. Lunardi CC, Moreira CM, Santos DL. Colesterolemia, trigliceridemia e excesso de peso em escolares de Santa Maria, RS, Brasil. Rev Bras Med Esporte [periódico na internet]. 2010 Ago [citado 2014 Maio 15]; 16(4): 250-253.

Disponível em: http://www.scielo.br/scielo.php?script=sci_arttext\&pid=\$1517-86922010000400003\&lng=en.

doi: 10.1590/S1517-86922010000400003.

25. Chiang-Salgado MT, Casanueva-Escobar V, Cid-Cea X, González-Rubilar U, Olate-Mellado P, Nickel-Paredes $F$ et al Factores de riesgo cardiovascular en estudiantes universitarios chilenos. Salud pública Méx [periódico na Internet]. 1999 Dez [citado 2014 Maio 19]; 41(6): 444-51. Disponível em: http://www.scielosp.org/scielo.php?script=sci_ arttext\&pid=S0036-36341999000600003\&lng=pt. doi: 10.1590/S0036-36341999000600003.

26. Toral N, Slater B, Silva MV. Consumo alimentar e excesso de peso de adolescentes de Piracicaba, São Paulo. Rev Nutr [periódico na Internet]. 2007 Out [citado 2014 Maio 15]; 20(5): 449-59. Disponível em: http://www.scielo.br/scielo. php?script=sci_arttext\&pid=S1415-52732007000500001\&lng=en. doi: 10.1590/S1415-52732007000500001.

27. Anjos LA. Índice de massa corporal (massa corporal.estatura-2) como indicador do estado nutricional de adultos: revisão da literatura. Rev Saúde Pública [periódico na internet]. 1992 Dez [citado 2014 Maio 12]; 26(6): 431-36. Disponível em: http://www. sceilo.br/scielo.php?script=sci_arttext\&pid=S0034-89101992000600009\&Ing=en. doi: 10.1590/S0034-89101992000600009 28. Piasetzki CTR; Bracht CM; Busnello MB; Winkelmann ER; Boff ETO; Franz LBB. Consumo Alimentar e Prática de Atividade Física em Estudantes Universitários. Revista Biomotriz, 2012; 6(1).

29. Cimadon HMS; Geremia R; Pellanda LC. Hábitos Alimentares, e Fatores de Risco para a Ateroesclerose em Estudantes de Bento Gonçalves (RS). Arq Bras de Cardiol, 2010; 95(2); 166-72. 
30. Berleze A, Haeffner LSB, Valentini NC. Prevalência de Obesidade na Infância em Diferentes Agrupamentos Sociais e a Importância de Estratégias Pedagógicas. Rev Saúde, 2008; 34(2); 44-9.

31. Mahan KL, Escott-Stump S. Alimentos Nutrição \& Dietoterapia. 11ª Edição. São Paulo: Roca; 2005.

32. Mendonça CP, Anjos LA. Aspectos das práticas alimentares e da atividade física como determinantes do crescimento do sobrepeso/obesidade no Brasil. Cad Saúde Pública [periódico na internet]. 2004 Jun [citado 2014 Maio 19]; 20(3): 698-709. Disponível em: http://www.scielo.br/scielo.php?script=sci_arttext\&pid=S0102-311X2004000300006\&lng=en. doi: 10.1590/S0102-311X2004000300006.

33. III Diretrizes Brasileiras Sobre Dislipidemias e Diretriz de Prevenção da Aterosclerose do Departamento de Aterosclerose da Sociedade Brasileira de Cardiologia. Arq Bras Cardiol. [periódico na internet]. 2001 Nov [citado 2014 Maio 15]: 1-48. Disponível em: http://www.scielo.br/scielo.php?script=sci_arttext\&pid=S0066-782X2001001500001\&lng=en. doi: 10.1590/S0066-782X2001001500001.

34. Godoy MF, Lucena JM, Miquelin AR, Paiva FF, Oliveira DLQ, Augustin JJL et al. Mortalidade por doenças cardiovasculares e níveis socioeconômicos na população de São José do Rio Preto, estado de São Paulo, Brasil. Arq. Bras. Cardiol. [periódico na internet]. 2007 Fev [citado 2014 Maio15]; 88(2): 200-206.

Disponível em: http://www.scielo.br/scielo.php?script=sci_arttext\&pid=S0066-782X2007000200011\&lng=en. doi: 10.1590/ S0066-782X2007000200011.

\section{Nilce Coelho Peixoto}

Endereço para correspondência - Universidade Federal de Santa Maria, Centro de Educação Superior Norte do Rio Grande do Sul - Campus Palmeira das Missões, Departamento de Ciências da Saúde, Av. Independência, n 3751 - Bairro: Vista Alegre, CEP: 98.300-000 - Palmeira das Missões - RS, Brasil E-mail: ncpeixoto@yahoo.com.br Lattes: http://lattes.cnpq.br/6573904661865745

Andressa Carine Kretschmer - kretschmerandressa@gmail.com

Géssica de Oliveira Rodrigues - geh_nutri@hotmail.com

Andressa dos Santos Ristow - dessa_ristow@hotmail.com

Enviado em 14 de outubro de 2014. Aceito em 13 de agosto de 2015. 